\title{
Ciclo Avaliação-Ação: Uma Abordagem para Análise do Desempenho em Matemática de Estudantes do Ensino Fundamental
}

\author{
Ricardo Augusto Lima de Souza - PPGECIM - ICE - UFAM \\ ricardo_souza@ufam.edu.br \\ José Francisco de Magalhães Netto - PPGECIM/PPGI - IComp - UFAM \\ jnetto.icomp@ufam.edu.br \\ Euler Vieira da Silva - PPGI - IComp - UFAM \\ eulervieira@ifam.edu.br
}

\section{Resumo}

Este artigo descreve a proposta do Ciclo Avaliação-Ação, uma abordagem para avaliação do processo de ensino-aprendizagem usando recursos computacionais de baixo custo e disponíveis para maioria dos professores. Como Estudo de Caso e fundamentado nos descritores da Prova Brasil, aplicaram-se testes simulados de Matemática para estudantes do $9^{\circ}$ Ano do Ensino Fundamental, tabulando os dados em Planilhas Eletrônicas para análise dos resultados de cada teste. Calcularam-se estatísticas dos desempenhos individualizados e das turmas, identificando questões com descritores e caracterizando o grau de domínio. Os relatórios forneceram as informações para o professor organizar intervenções pedagógicas focadas nos descritores com maior grau de dificuldade, acelerando o ciclo ensino-aprendizagem-avaliação.

Palavras-Chave: Avaliação, Educação Matemática, Desempenho, Planilha Eletrônica, Prova Brasil.

\section{An Approach to Analysis Mathematics Performance Students of Fundamental School}

\section{Abstract}

This paper describes the proposal of the Assessment-Action Cycle, an approach to evaluate the teaching-learning process using low-cost computing resources available to most teachers. As a case study and based on the descriptors of Prova Brasil, we applied simulated Mathematics tests to students of the 9th Year of Basic Education, tabulating the data in Spreadsheets to analyze the results of each test. Statistics of individualized performances and classes were calculated, identifying the questions with descriptors and characterizing their degree of mastery. The reports provided the information for the teacher to organize pedagogical interventions focused on the descriptors with greater difficulty, accelerating the teaching-learning-evaluation cycle.

Key-words: Assessment, Mathematics Education, Performance, Spreadsheet, Prova Brasil.

\section{Introdução}

Na escola pública de Ensino Fundamental as Tecnologias Digitais da Informação e Comunicação (TDIC) constituem uma possibilidade de prática pedagógica dos professores (BORBA, 2001). Dessa forma, identifica-se em determinados contextos e fases, a integração da avaliação escolar inserida no cotidiano das escolas do Ensino Fundamental com auxílio das tecnologias para avaliação e identificação do nível de aprendizagem dos estudantes (BORBA; SILVA; GADINIDIS, 2014). 
Nos sistemas educacionais brasileiros os Parâmetros Curriculares Nacionais (PCNs) formam a base para avaliação na Matemática Escolar com foco central na Resolução de Problemas, que possibilita aos professores orientações básicas sobre o ensino desta disciplina (BRASIL, 1997). Neste contexto, os sistemas de avaliações externas em larga escala, que objetivam elaborar diagnósticos dos sistemas educacionais (HORTA NETO, 2007). Adicionalmente, no quadro de avaliações educacionais brasileiras, insere-se o Sistema de Avaliação da Educação da Básica (SAEB), coordenado pelo Instituto Nacional de Estudos e Pesquisas Educacionais Anísio Teixeira (INEP), órgão vinculado ao Ministério da Educação (MEC).

O SAEB tem entre seus objetivos "buscar o desenvolvimento de uma cultura avaliativa que estimule o controle social sobre os processos e resultados" (BRASIL, 2008, p. 8). Também, examina a proficiência dos alunos, gerando indicadores educacionais. Assim, nesse processo os resultados das avaliações auxiliam a formulação de políticas públicas educacionais dos governos municipais, estaduais e federal.

A partir de sua reforma em 2005, o SAEB tornou-se censitário e atualmente reúne três avaliações, a Avaliação Nacional da Educação Básica (ANEB), o Sistema Nacional de Alfabetização (ANA), e a Avaliação Nacional do Rendimento Escolar (ANRESC) compõem sistema o avaliativo brasileiro (BRASIL, 2008).

Por seu turno, a ANRESC é mais conhecida como Prova Brasil que é censitária para as escolas públicas do Ensino Fundamental. Além do mais, a Prova Brasil gera resultados do desempenho educacional em Português e Matemática de escolas, com quantidade no mínimo de vinte estudantes matriculados no $5^{\circ}$ Ano e no $9^{\circ}$ Ano do Ensino Fundamental. Desta forma, os dados gerados e analisados possibilitam os debates e a elaboração de políticas educacionais para melhorar a proficiência dos estudantes (BRASIL, 2008).

Os resultados divulgados do período entre 1995 e 2015 na avaliação de Matemática dos estudantes da $9^{\circ}$ ano de escolas urbanas, revelam maior concentração de alunos nos níveis mais baixos da escala de proficiência (INEP, 2016).

Igualmente, estes dados revelam que a proficiência média em Matemática ficou em 256 pontos dos estudantes avaliados pela Prova Brasil 2015, onde a pontuação máxima é 425 pontos. Esta média situa-se na escala de proficiência no nível três de um total de nove níveis, ou seja, a grande maioria não atingiu o nível adequado de proficiência nesta disciplina (INEP, 2016).

Em geral, tem-se realizado a Prova Brasil no mês de novembro dos anos ímpares sob a forma impressa. Entretanto, o tempo entre a aplicação, a análise dos resultados e o envio dos relatórios para os professores e gestores das escolas das Secretarias de Educação é longo, durando cerca de seis meses após a aplicação, tornando o processo moroso e pouco efetivo.

Para a preparação das avaliações externas, as escolas brasileiras geralmente aplicam simulados impressos com o objetivo de melhorar o desempenho dos seus alunos. Normalmente, resultados dos simulados não são organizados e disponibilizados aos professores e gestores das escolas na forma de relatórios estatísticos.

Diante desse quadro, a pesquisa busca diminuir o intervalo de tempo entre a aplicação, análise e divulgação dos resultados oficiais, possibilitando aos professores e gestores envolvidos acessarem os relatórios gerados em tempo hábil com base nas avaliações e consigam aplicar intervenções pedagógicas no seu cotidiano escolar. Para 
ser alcançada esta agilidade, propomos nessa pesquisa o uso de TDIC, em particular, Planilhas Eletrônicas, que foi a ferramenta escolhida dada a natureza desse trabalho.

Dessa maneira, aponta-se a seguinte pergunta de pesquisa: qual o nível do desempenho em simulados de Matemática de Estudantes do $9^{\circ}$ Ano do Ensino Fundamental de uma Escola Pública com a abordagem do Ciclo Avaliação-Ação e o uso de Planilha Eletrônica pelo professor?

Diante disso, o nosso objetivo de pesquisa é analisar o nível do desempenho em simulados de Matemática de estudantes do $9^{\circ}$ Ano do Ensino Fundamental de uma Escola Pública com a abordagem Ciclo Avaliação-Ação em conjunto com uso de Planilha Eletrônica pelo professor.

Para relatar a pesquisa, este artigo divide-se em cinco seções, incluindo a introdutória, compondo-se de, na ordem, em: Trabalhos Correlatos, Metodologia, Análise e Discussão de Resultados e as Conclusões, bem como as limitações, contribuições e as sugestões para trabalhos futuros.

\section{Trabalhos correlatos}

A abordagem pedagógica desenvolvida integra-se no processo ensino-aprendizagemavaliação. Assim, centra-se nesse processo, pois auxilia o professor nas atividades escolares. Na avaliação escolar do Ensino Fundamental registra-se o grau de aprendizagem dos alunos, pois o objetivo é examinar o nível de aprendizado, particularmente na aprendizagem da Matemática.

Perrenoud (2001) propõe que as habilidades e competências servem para resolver situações com a abordagem de aprendizagem em conjunto com o professor na aplicação sistemática do modelo de administração de progressão de aprendizagens.

Kilpatrick (2001) desenvolveu um estudo sobre proficiência matemática que se constitui por cinco elementos interdependentes e simultâneos. Estes elementos são: compreensão de conceitos, fluência de procedimentos, elementos de competência de estratégias, raciocínio adaptativo e disposição para a produtividade.

Bonamino e Souza (2011) descreveram a linha do tempo e o processo de evolução do SAEB, desde o início em 1990 até 2012. Na elaboração dessa avaliação externa adotou-se a Teoria de Resposta ao Item (TRI), pois comparou os resultados de várias áreas do conhecimento no decorrer dos anos que resulta no histórico das avaliações em uma linha de tempo.

Em adição, o INEP que coordena a Prova Brasil de Matemática estabeleceu o eixo norteador da avaliação centrada na Resolução de Problemas, para aumentar os significados com uso de situações desafiadoras. A Resolução de Problemas está associada ao desenvolvimento de capacidades como observação, estabelecimento de relações, comunicação de diferentes linguagens, argumentação e validação de processos, além de estimular formas de raciocínio como intuição, indução, dedução e estimativa.

Houve uma revisão em 2001 das matrizes de referência da Prova Brasil, reunindo os conteúdos em tópicos ou temas e as descrições das habilidades e descritores, para avaliar cada área do conhecimento especificamente no $5^{\circ}$ Ano e também no $9^{\circ}$ Ano do Ensino Fundamental (BRASIL, 2008).

Adicionalmente, no documento oficial Brasil (2008) definiu descritor como uma associação entre conteúdos curriculares e operações mentais desenvolvidas pelo estudante, que traduzem competências e habilidades. Os descritores apontariam as 
habilidades gerais que se esperam dos alunos, formulando a referência para seleção das questões que devem compor uma prova de avaliação.

Basso (2015) em estudo abordando o impacto do uso de recursos digitais que oferecem a possibilidade de operar com Matemática estão em uma curva ascendente na literatura da Informática na Educação e na Educação Matemática. Segundo o autor em um levantamento das publicações tratando de Matemática na Revista Brasileira de Informática na Educação (RBIE) de 1999 a 2014, identificou 17 trabalhos publicados de 1999 a 2011 e 19 trabalhos de 2012 a 2014, evidenciando o crescimento nas produções e o interesse nessa temática. Igualmente, o tema aparece em publicações da Revista de Novas Tecnologias na Educação (RENOTE). No período de 2003 a 2015, 183 artigos foram publicados sobre tecnologias e Matemática.

Lipp, Mossmann e Bez (2014) propõem pesquisas para a melhoria do ensino de Matemática com uso de Novas Tecnologias, em particular usando objetos de aprendizagem, após os resultados recentes da baixa classificação dos estudantes em avaliações

em larga escala, como a Prova Brasil e o Programa Internacional de Avaliação de Estudantes (PISA - Programme for International Student Assessment).

Pinheiro (2013) relatou uma aplicação de ferramenta que serve como instrumento de auxílio aos professores e gestores no dia a dia escolar, com base nas avaliações externas com foco na Prova Brasil do INEP.

Com o propósito de ilustrar o uso da tecnologia aplicada ao Ensino de Matemática Borba, Silva e Gadinidis (2014) esquematizaram a organização das tecnologias digitais em fases. Destacaram o surgimento de uma fase por meio das inovações tecnológicas que possibilitaria a organização de cenários qualitativos diferenciados de investigação matemática, aplicando prática pedagógica usando novo recurso tecnológico original ao pensar-com-tecnologia.

Farias (2013) analisou o uso do computador no processo de avaliação aplicado a alunos do $5^{\circ}$ Ano do Ensino Fundamental associado ao uso da TRI no processo de avaliação e obtenção de resultados. $\mathrm{O}$ autor propôs o desenvolvimento de um módulo de avaliação online que possibilitasse a realização de um teste no computador. Foi aplicado um teste impresso com o uso da TRI e também na forma digital. A seguir, avaliou os resultados, elaborando levantamento das vantagens e desvantagens de aplicar o computador no processo de avaliação.

Igualmente, Venâncio e Lopes (2012) descreveram a implementação do protótipo de um sistema interativo de gestão de avaliação processual baseado em competências e ressaltam a importância da avaliação formativa, apontada pelo currículo contemporâneo no ambiente escolar. Os autores relataram o desenvolvimento do protótipo inicial e, por fim, apresentaram resultados da sua avaliação realizada com educadores do Ensino Fundamental.

Em suma, a literatura sobre Matemática e também de Tecnologias aplicadas à Educação é abrangente. Entretanto, a contribuição diferenciada dessa pesquisa é a análise do desempenho em Matemática do $9^{\circ}$ Ano do Ensino Fundamental com a abordagem do Ciclo Avaliação-Ação com o emprego de Planilha Eletrônica. 


\section{Metodología}

O estudo classifica-se como uma pesquisa descritiva, pois segundo Gil (2002), a pesquisa descritiva descreve as características de uma população. Outra finalidade é a identificação de possíveis relações entre as variáveis.

A pesquisa classifica-se como quali-quanti. No objetivo qualitativo, examina a questão de pesquisa, ou seja, a interação entre variáveis e processos dinâmicos. No objetivo quantitativo, aplica coleta e tratamento de dados com estatística não aprofundando as causas e precisão dos resultados, evitando distorções de análise e interpretação, aplicável em pesquisas descritivas.

A metodologia de Estudo de Caso permite a exploração de um 'sistema limitado', no tempo e em profundidade, através de uma coleta de dados profunda envolvendo fontes múltiplas de informação no contexto (COUTINHO, 2014; RAMOS, e NARANJO, 2014). Nesse caso, conforme Yin (2010), deve-se constituir como estratégia de investigação de uma situação contemporânea. É um tipo de investigação que permite a realização de um exame sistemático, detalhado, intensivo e interativo das dificuldades e possibilidades.

Para organizar esta pesquisa usou-se, portanto, um Estudo de Caso, que permite relatar a ação realizada na prática em sala de aula e analisá-la com as descrições e interpretações dos conteúdos baseando-se nos documentos oficiais do SAEB/INEP na observação participante e nas entrevistas. Em seguida houve a apresentação do projeto e discussão para participação com professora regente da turma. A pesquisa seguiu os passos mostrados na Figura 1, que denominamos Ciclo Avaliação-Ação:

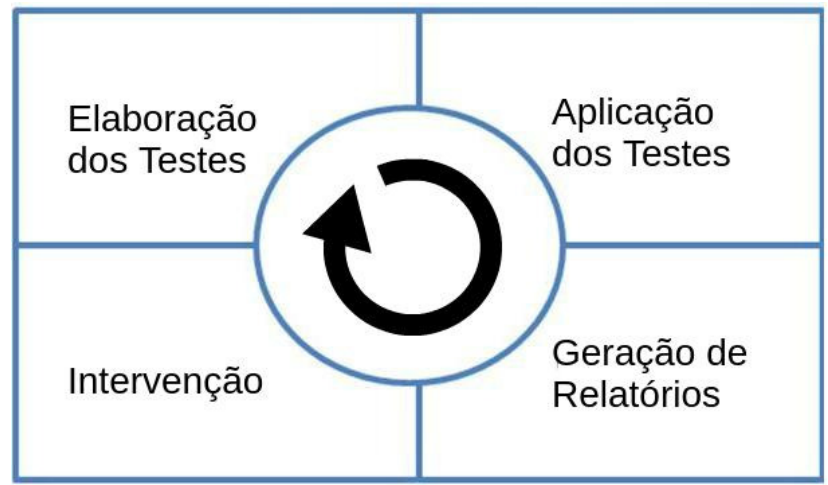

Figura 1 - Representação do Ciclo Avaliação-Ação

Primeiro, organizou-se um banco de questões similares às questões usadas na Prova Brasil de acordo com os descritores de Matemática para o $9^{\circ}$ Ano do Ensino Fundamental do documento do SAEB/INEP (INEP, 2011).

Deste banco, esquematizou-se um conjunto de questões para aplicar nas sessões avaliativas que continham dez questões. Geraram-se relatórios da turma e individuais dos estudantes com tabelas e gráficos. Ajustou-se o tempo de resolução da prova em relação ao nível das questões, em seguida estabeleceram-se o grau de acerto maior que cinquenta por cento para aprovação do estudante.

No segundo momento, definiu-se a escala de desempenho com distribuição das notas para identificar os relatórios estatísticos associados a cada nota. Então, classificaram-se as questões em três níveis, básico, intermediário e difícil. Finalmente as questões foram escolhidas em função das matrizes adotadas pelo SAEB com os 
respectivos temas e descritores. Os temas são: Espaço e Forma, Grandezas e Medidas, Números e Operações/Álgebra/Funções e Tratamento da Informação.

Embora esses temas escolhidos não contemplem todos os objetivos educacionais de Matemática Básica (BRASIL, 2008), mas apenas aqueles considerados relevantes e possíveis de serem mensurados em uma avaliação para, com isso, obter-se informações que forneçam uma visão real do nível de aprendizagem dos alunos e da turma, já que os descritores indicam as habilidades a serem avaliadas em cada tema.

No terceiro momento, os estudantes realizaram as sessões de testes simulados. Em cada sessão empregou-se dez questões de Matemática do $9^{\circ}$ Ano do Ensino Fundamental, que foram respondidas em cinquenta minutos. A sessão iniciou-se com a distribuição dos testes e foram dadas instruções básicas para sua realização. Após o término, todos os estudantes assinalaram as respostas no cartão, conforme estipulado pelas regras do INEP. Em seguida, as respostas foram tabuladas em uma Planilha Eletrônica. Em seguida apresentaram-se os resultados para a professora, que analisou e planejou as intervenções pedagógicas.

Durante a aplicação da abordagem a professora acessou os relatórios para análises das etapas das avaliações e aplicação das intervenções. Depois, esquematizouse outro conjunto de questões para outras sessões avaliativas, também compostas de treze a vinte e seis questões. Em seguida, no quarto momento, os alunos participaram das intervenções pedagógicas e atividades sugeridas pela professora com o objetivo de melhorar o grau de proficiência, com base nos relatórios individualizados e por turma.

Consequentemente, o ciclo de aplicação das sessões avaliativas nas turmas do $9^{\circ}$ Ano, foi novamente aplicado gerando relatórios de desempenho individuais de erros e acertos e relatórios de desempenho das turmas. Em consequência, propuseram-se as intervenções pedagógicas focadas com base nos resultados estatísticos dos relatórios individuais e também das turmas.

Posteriormente, analisaram-se os erros mais frequentes para aplicar as intervenções pedagógicas com base nos descritores com maior índice de erros. A seguir, aplicaram-se práticas para aumentar o grau de acertos, finalmente resultando em intervenções pedagógicas eficazes.

\section{Análise e Discussão de Resultados}

A pesquisa foi realizada com aplicação de quatro testes na forma impressa, com a participação de duas turmas com aproximadamente setenta estudantes do $9^{\circ}$ Ano do Ensino Fundamental de uma Escola Pública estadual da cidade de Manaus (AM), sob a regência de uma professora entre os meses de agosto e novembro de 2015.

Os estudantes já participavam de simulados periódicos realizados na escola com base nos critérios propostos pelo INEP. A prática de avaliação alinhou-se com as metas educacionais da escola que usou simulados semelhantes às questões oficiais.

Após as avaliações, os dados foram tabulados na Planilha Eletrônica, onde geraram-se relatórios de desempenho individuais e por turmas, apontando uma visão abrangente dos desempenhos, permitindo a professora, traçar estratégias das intervenções pedagógicas. A Figura 2 ilustra o exemplo de uma questão aplicada na avaliação, do bloco de treze questões. Em particular, a questão associa-se aos descritores D21 e D25; D21 que avalia a habilidade do estudante de identificar números racionais nas suas diversas representações: fracionária, decimal ou percentual e o D25 
que avalia a habilidade efetuar cálculos que envolvam operações com números racionais.

7. Se $b=0,111 \ldots$ e $a=0,555 \ldots, a-b$ pode
ser representado por:
$\begin{array}{llll}\text { a) } \frac{2}{3} & \text { b) } \frac{6}{10} & \text { c) } \frac{2}{9} & \text { d) } \frac{4}{9}\end{array}$

Figura 2 - Enunciado de Questão dos descritores D21e D25 Fonte: Autor.

A Figura 3 ilustra a planilha de resultados tabulados em duas partes. A primeira parte faz a identificação dos estudantes e apresenta o registro das respostas de uma avaliação. A segunda parte registra o número de erros e acertos dos estudantes por questão, além de mostrar a totalização dos acertos.
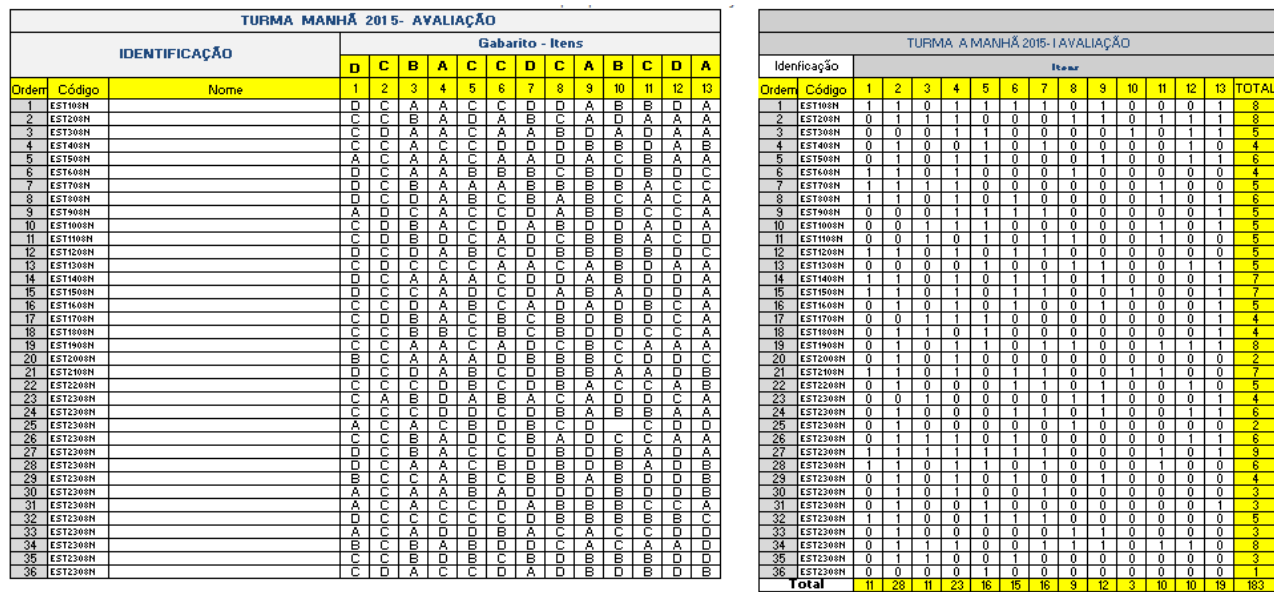

Figura 3 - Visão Geral da Planilha Eletrônica aplicada nas avaliações Fonte: Autor.

O resultado em forma de gráfico de barras e de setores gerados na décima segunda semana da pesquisa está apresentado na Figura 4, onde registram-se o aproveitamento da turma por questão. As totalizações das respostas dos estudantes para cada alternativa, neste caso particular relativa à questão 07. Acrescenta-se que a visualização da resposta certa possibilitou à professora fazer uma comparação imediata da quantidade de acertos para identificar o grau de aproveitamento da turma.

\begin{tabular}{|c|c|c|}
\hline Alternativas & Respostas dos estudantes & Gabarito \\
\hline A & 9 & \\
\hline B & 9 & \\
\hline C & 2 & \\
\hline D & 16 & Certo \\
\hline
\end{tabular}

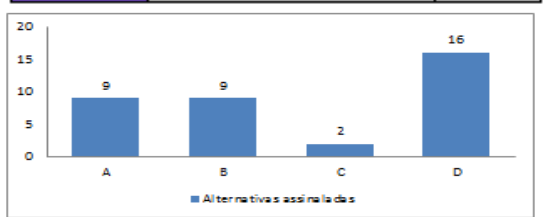

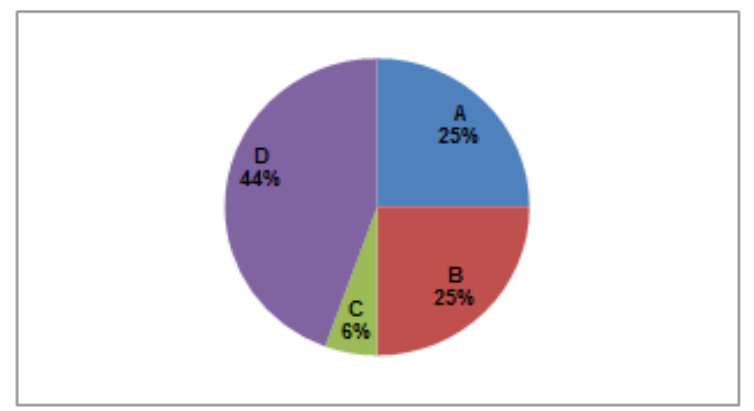

Figura 4 - Aproveitamento da Turma por Questão Fonte: Autor. 
As porcentagens das respostas da questão 07 ilustram-se na Figura 5, na forma de gráfico de setores e uma tabela que expressa à distribuição das alternativas escolhidas pelos estudantes. Com base na informação o professor pode analisar com abrangência, pois, a representação percentual facilita a visualização das respostas.

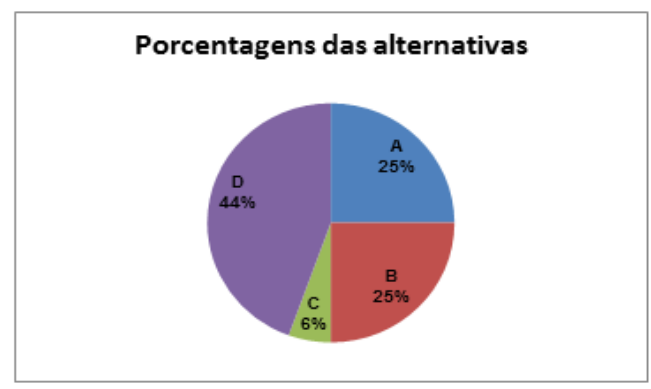

\begin{tabular}{|l|c|c|c|c|}
\hline \multicolumn{5}{|c|}{ Repostas dos estudantes } \\
\hline Alternativas & A & B & C & D \\
\hline Porcentagens & $25 \%$ & $25 \%$ & $6 \%$ & $44 \%$ \\
\hline
\end{tabular}

Figura 5 - Aproveitamento da Turma por Questão em porcentagens Fonte: Autor.

Em particular, o gráfico de barras do desempenho individual de um determinado estudante na segunda avaliação mostrado na Figura 6, permite ao professor identificar os resultados para cada estudante com o respectivo nível de proficiência em Matemática, conforme a questão e o descritor.

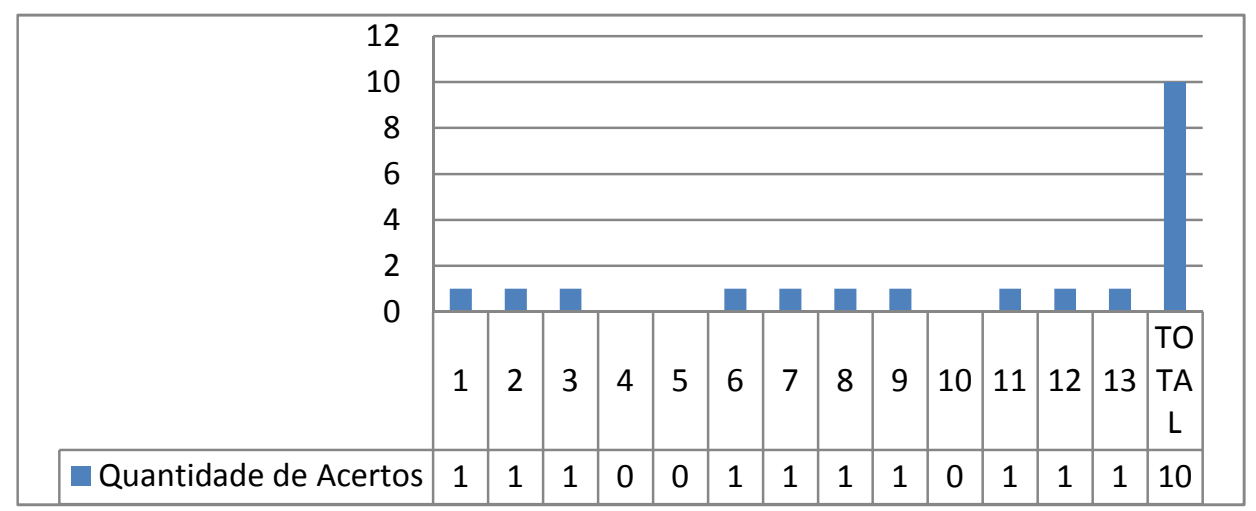

Figura 6 - Aproveitamento Individual por Questão

Fonte: Autor.

Por fim, aplicou-se uma entrevista com a professora participante da pesquisa para investigar a validação da abordagem. A professora que participou da experiência relatou contribuições positivas da pesquisa em sua prática pedagógica e dentre essas contribuições, destacou o acompanhamento individual e por turma dos relatórios gerados pela Planilha Eletrônica que permitiram um detalhamento objetivo para o planejamento das intervenções pedagógicas, fornecendo um minucioso panorama do desempenho individual e da turma.

Uma limitação da abordagem foi o não aprofundamento do uso de Estatística Descritiva para aplicação nos resultados dos relatórios de desempenhos dos estudantes. Dessa forma, ilustrariam os resultados com tratamento estatístico de médias e desviospadrão que permitem mais detalhamento e objetividade.

Ao optarmos por uma solução de baixo de custo, abrimos mão de soluções mais sofisticadas empregadas como, por exemplo, a solução baseada em Learning Analytics, conforme adotado na pesquisa sobre visualização de desempenhos (SILVA; NETTO; 
SOUZA, 2016). Soluções mais elaboradas exigem sistemas computacionais caros e também envolvem custo de manutenção.

Dadas as especificidades da Região Amazônica onde o acesso à Internet ainda é precário, optou-se por solução que não dependa disso. Acrescenta-se que a maioria dos professores usuários de computadores da região não possui acesso à Internet. Por outro lado, a solução adotada além de ser de baixo custo por não depender de sistema específico e nem da Internet e por ser baseada em Planilha Eletrônica, permite um vinculo maior do professor, deste modo torna o envolvimento mais rápido do mediador proporcionando autonomia e liberdade para atuar em sala de aula. Portanto, o mediador torna-se a agente central da abordagem.

\section{Conclusões}

Desta forma, alcançou-se o objetivo de descrever a abordagem Ciclo Avaliação-Ação de com o uso de Planilha Eletrônica para análise pelo professor do nível de desempenho em simulados de Matemática de Estudantes do $9^{\circ}$ Ano do Ensino Fundamental de uma Escola Pública. Destacadamente, a professora usou os relatórios gerados para organizar as intervenções pedagógicas individuais dos estudantes e das turmas, centradas nos descritores que apontaram maior porcentagem de erros.

Para concretização dessa abordagem os recursos usados são relativamente baratos e acessíveis, pois é necessário apenas o uso de um computador e conhecimentos básicos em informática pelos professores ou gestores. Da mesma forma não é necessário acesso à Internet para realizar as tabulações dos resultados. O ciclo com completou-se num intervalo de duas a três semanas, dando dinamismo ao processo avaliativo.

A abordagem do Ciclo Avaliação-Ação com o uso de Planilhas Eletrônicas por professores de Escolas Públicas resulta em benefícios didáticos. Em particular, apontase o elevado benefício mensurado pelo baixo custo. Especificamente, o custo de alguns aplicativos é gratuito, pois estão instalados nos computadores distribuídos aos professores de Ensino Fundamental do Estado, registrando fator de impacto socioeconômico. Assim, a relevância social da pesquisa reflete-se na melhoria das avaliações com o uso de computadores e programas de baixo custo.

A abordagem adotada pode ser aplicada em outras áreas de ensino da Matemática e, em particular, para situações em que o processo de ensino-aprendizagemavaliação baseia-se na solução de problemas, como é o caso das áreas de Ciências Exatas, por exemplo, Química e Física.

Como trabalhos futuros pretende-se ampliar a quantidade de turmas e experimentos e também aplicar a abordagem no intervalo do $6^{\circ}$ ao $9^{\circ}$ Anos do Ensino Fundamental. Salienta-se que o aperfeiçoamento está em andamento com base em sugestões apontadas pela colaboradora e equipe. Como trabalhos futuros pretende-se incrementar novas funcionalidades tais como cálculo de médias e desvio-padrão. Por fim, a relevância socioeconômica da pesquisa reflete-se na melhoria das avaliações com o uso de computadores e programas de baixo custo. 
Referências

BASSO, M.; NOTARE, M R. Pensar-com Tecnologias Digitais de Matemática Dinâmica. Revista Novas Tecnologias na Educação (RENOTE). Porto alegre, v. 13, n. 2, 2015.

BONAMINO, A.; SOUSA, S. Z. Três Gerações de Avaliação da Educação Básica. Educação e Pesquisa, v. 38, n. 2, p. 373-388. 2012

BORBA, M. de C.; PENTEADO, M. G. Informática na Educação. Belo Horizonte: Autêntica Editora, 2001.

BORBA, M. C.; SILVA, R. S. R.; GADINIDIS, G. Fases das Tecnologias Digitais em Educação Matemática: Sala de Aula e Internet em Movimento. $1^{\text {a }}$ ed. - Belo Horizonte: Autentica Editora, 2014.

BRASIL. Ministério da Educação. PDE: Plano de Desenvolvimento da Educação: Prova Brasil: Brasília : MEC, SEB; Inep, 2008.

Parâmetros Curriculares Nacionais. Brasília, DF, 1997.

COUTINHO, P. C. Metodologia de Investigação em Ciências Sociais e Humanas: Teoria e prática. Editora Almedina, 2014.

FARIAS, A. C. M. O. Aplicação da Teoria de Resposta ao Item na Avaliação Educacional e seu Processo de Informatização - Dissertação de Mestrado, UFMA, São Luís, 2013.

GIL, A. C. Como Elaborar Projetos de Pesquisa. - 4. ed. - São Paulo: Atlas.

HORTA NETO, J. L. Um Olhar Retrospectivo Sobre a Avaliação Externa no Brasil: das Primeiras Medições em Educação até o SAEB de 2005. Revista Iberoamericana de Educación nº42/5, 2007.

INEP. Instituto Nacional de Estudos e Pesquisas Educacionais Anísio Teixeira Prova Brasil. Resumo dos Resultados SAEB 2015. Brasília, DF, 2016.

KILPATRICK, J.; SWAFFORD, J.; AND FINDELL, B. Adding It Up: Helping Children Learn Mathematics, National Academy Press, Washington, DC, 2001.

LIPP, M. K; MOSSMANN, J. B.; BEZ, M. R. Desenvolvimento de objetos de aprendizagem para a Matemática utilizando o dispositivo de NUI Leap Motion. Revista Novas Tecnologias na Educação (RENOTE). Porto Alegre, v. 12, n. 2, 2014.

PERRENOUD, P. Dez Novas Competências para Ensinar. Porto Alegre: Artmed Editora, 2000.

PINHEIRO, R. G. P.; ELIA, M., \& SAMPAIO, F. F.. Avaliando as Competências Escolares Através da Prova Brasil Usando Ferramenta Web. In XIX Workshop de Informática na Escola (WIE). Campinas-SP, 2013.

RAMOS, S. T. C. \& NARANJO, E. S. Metodologia da investigação científica. Editora Escolar, 2014.

SILVA, E. V., NETTO. J.F.M., SOUZA, R.A.L. O Uso de Dashboard na Identificação do Desempenho de Alunos de Matemática Básica. In TISE XXI Congreso Internacional de Informática Educativa. Santiago - Chile, 2016.

VENÂNCIO, V.; LOPES, R. DE D. Competências e Avaliação Formativa em Sistema Interativo de Apoio a Aprendizagem e ao Planejamento voltado ao Ensino Fundamental. In: Anais dos Workshops do Congresso Brasileiro de Informática na Educação, 2012.

YIN, R. K. Estudo de caso: planejamento e métodos. Editora Artmed, 2010. 